\title{
A group-theoretic viewpoint on Erdös-Falconer problems and the Mattila integral
}

\author{
Allan Greenleaf, Alex Iosevich, Bochen Liu and Eyvindur Palsson
}

\begin{abstract}
We obtain nontrivial exponents for Erdős-Falconer type point configuration problems. Let $T_{k}(E)$ denote the set of distinct congruent $k$-dimensional simplices determined by $(k+1)$-tuples of points from $E$. For $1 \leq k \leq d$, we prove that there exists a $t_{k, d}<d$ such that, if $E \subset \mathbb{R}^{d}$, $d \geq 2$, with $\operatorname{dim}_{\mathcal{H}}(E)>t_{k, d}$, then the $\left(\begin{array}{c}k+1 \\ 2\end{array}\right)$-dimensional Lebesgue measure of $T_{k}(E)$ is positive. Results of this type were previously obtained for triangles in the plane $(k=d=2)$ in [8] and for higher $k$ and $d$ in [7]. We improve upon those exponents, using a group action perspective, which also sheds light on the classical approach to the Falconer distance problem.
\end{abstract}

\section{Introduction}

One of the most important and far reaching problems in modern geometric measure theory is the Falconer distance problem, which asks: How large does the Hausdorff dimension $s$ of a compact set $E \subset \mathbb{R}^{d}, d \geq 2$, need to be to ensure that the distance set of $E, \Delta(E):=\{|x-y|: x, y \in E\} \subset \mathbb{R}$, has positive Lebesgue measure? Falconer proved that $s>d / 2$ is necessary, up to the endpoint, and conjectured that it is also sufficient [6]. The best exponent known to date is $d / 2+1 / 3$, due to Wolff in the plane [15] and Erdog̃an in higher dimensions [3].

A natural extension of the Falconer distance problem is the congruent simplex problem [4], [8], [7]. We say that $\left\{x^{1}, \ldots, x^{k+1}\right\} \subset \mathbb{R}^{d}$ is nondegenerate (or affinely independent) if $\left\{x^{2}-x^{1}, x^{3}-x^{1}, \ldots, x^{k+1}-x^{1}\right\}$ is linearly independent. This condition is of course invariant under permutations, and is equivalent with the convex hull of $\left\{x^{1}, \ldots, x^{k+1}\right\}$ having positive $k$-dimensional volume and thus being reasonably called the $k$-simplex generated by $x^{1}, \ldots, x^{k+1}$, denoted $\Delta\left(x^{1}, \ldots, x^{k+1}\right)$.

Given a set $E \subset \mathbb{R}^{d}$, let $E^{k+1}:=E \times E \times \cdots \times E,(k+1)$-times.

Definition 1.1. Let $d \geq 2$ and $1 \leq k \leq d$. Given a set $E \subset \mathbb{R}^{d}$, define the set of distinct congruent simplices determined by $E$ to be $T_{k}(E):=E^{k+1} / \sim$, where

Mathematics Subject Classification (2010): Primary 42B20; Secondary 52C10.

Keywords: Erdős-Falconer problems, Mattila integral. 
$\left(x^{1}, \ldots, x^{k+1}\right) \sim\left(y^{1}, \ldots, y^{k+1}\right)$ if and only if $\left\{x^{i}\right\}_{j=1}^{k+1}$ and $\left\{y^{i}\right\}_{j=1}^{k+1}$ are nondegenerate and $\left|x^{i}-x^{j}\right|=\left|y^{i}-y^{j}\right|$ for $1 \leq i<j \leq k+1$.

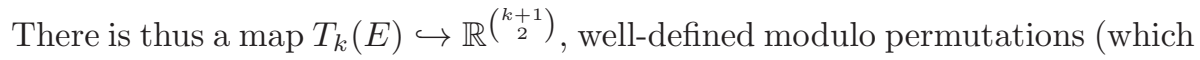
have no effect on positivity of Lebesgue measure and hence will be ignored),

$$
\left[\left(x^{1}, \ldots, x^{k+1}\right)\right] \longrightarrow\left(\left|x^{i}-x^{j}\right|\right)_{1 \leq i<j \leq k+1} .
$$

One may also consider similar simplices instead of congruent ones:

Definition 1.2. Given a compact set $E \subset \mathbb{R}^{d}$ define the set of distinct similar simplices determined by $E$ to be $S_{k}(E):=E^{k+1} / \sim$, where $\left(x^{1}, \ldots, x^{k+1}\right) \sim$ $\left(y^{1}, \ldots, y^{k+1}\right)$ if and only if $\left\{x^{i}\right\}_{j=1}^{k+1}$ and $\left\{y^{i}\right\}_{j=1}^{k+1}$ are nondegenerate and, for some $\lambda>0,\left|x^{i}-x^{j}\right|=\lambda\left|y^{i}-y^{j}\right|$, for $1 \leq i<j \leq k+1$.

By considerations similar to those for $T_{k}(E)$, one can view $S_{k}(E)$ as a subset of the projective space $\mathbb{R P} \mathbb{P}^{\left(\begin{array}{c}k+1 \\ 2\end{array}\right)-1}$ or, in local coordinates, $\mathbb{R}^{\left(\begin{array}{c}k+1 \\ 2\end{array}\right)-1}$.

In this paper, we obtain improved (i.e., reduced) lower bounds on the Hausdorff dimension of $E$ that guarantee that $T_{k}(E)$ and $S_{k}(E)$ are of positive $\left(\begin{array}{c}k+1 \\ 2\end{array}\right)$ and $\left(\begin{array}{c}k+1 \\ 2\end{array}\right)-1$ dimensional Lebesgue measure, resp. The central idea is a geometric mechanism for studying such problems based on group actions, a method that sheds some new light even on the classical approach to the Falconer distance problem. Our first two results contain the essential features of the method.

Theorem 1.3. Let $E$ be a compact set in $\mathbb{R}^{d}, d \geq 2$, and $\mu$ a finite, nonnegative measure supported on $E$. For $g \in \mathbb{O}(d)$, the orthogonal group on $\mathbb{R}^{d}$, define a measure $\nu_{g}$, supported on $E-g E$, by the relation

$$
\int_{\mathbb{R}^{d}} f(z) d \nu_{g}(z):=\int_{E} \int_{E} f(u-g v) d \mu(u) d \mu(v), \quad f \in C_{0}\left(\mathbb{R}^{d}\right) .
$$

Define also a measure $\nu$ on $T_{k}(E) \subset \mathbb{R}^{\left(\begin{array}{c}k+1 \\ 2\end{array}\right)}$ by

$$
\begin{aligned}
& \int f(\mathbf{t}) d \nu(\mathbf{t}) \\
& \quad=\int \ldots \int f\left(\left|x^{1}-x^{2}\right|, \ldots,\left|x^{i}-x^{j}\right|, \ldots,\left|x^{k}-x^{k+1}\right|\right) d \mu\left(x^{1}\right) \ldots d \mu\left(x^{k+1}\right),
\end{aligned}
$$

where the entries of the $\left(\begin{array}{c}k+1 \\ 2\end{array}\right)$-vector $\mathbf{t}$ are the distances $t_{i j}$ from $x^{i}$ to $x^{j}, 1 \leq i<$ $j \leq k+1$.

Then, if $\nu_{g}$ is absolutely continuous for a.e. $g \in \mathbb{O}(d)$, with density also denoted $\nu_{g}$, and

$$
\int_{\mathbb{O}(d)} \int_{\mathbb{R}^{d}} \nu_{g}^{k+1}(x) d x d g<\infty,
$$

where $d g$ is Haar measure on $\mathbb{O}(d)$, then the measure $\nu$ in $(1.2)$ has an $L^{2}$ density and $\mathcal{L}^{\left(\begin{array}{c}k+1 \\ 2\end{array}\right)}\left(T_{k}(E)\right)>0$. 
We obtain an analogous result for similarity classes.

Theorem 1.4. Let $E$ and $\mu$ be as in Theorem 1.3. For $a \in \mathbb{R}^{+}, g \in \mathbb{O}(d)$, define a measure $\nu_{a, g}$ by

$$
\int_{\mathbb{R}^{d}} f(z) d \nu_{a, g}(z):=\int_{E} \int_{E} f(u-a g v) d \mu(u) d \mu(v),
$$

Let $I \subset \mathbb{R}^{+}$be a a compact interval. Then, if $\nu_{a, g}$ is absolutely continuous for a.e. $(a, g) \in I \times \mathbb{O}(d)$ and

$$
\int_{I} \int_{\mathbb{O}(d)} \int_{\mathbb{R}^{d}} \nu_{a, g}^{k+1}(x) d x d g \frac{d a}{a}<\infty
$$

then $\mathcal{L}^{\left(\begin{array}{c}k+1 \\ 2\end{array}\right)-1}\left(S_{k}(E)\right)>0$.

As applications of Theorems 1.3 and 1.4, one obtains:

Theorem 1.5. Let $E \subset \mathbb{R}^{d}, d \geq 2$, and $2 \leq k \leq d$. Suppose that

$$
\operatorname{dim}_{\mathcal{H}}(E)>t_{k, d}:=\frac{d k+1}{k+1} .
$$

Then $\mathcal{L}^{\left(\begin{array}{c}k+1 \\ 2\end{array}\right)}\left(T_{k}(E)\right)>0$. If $d=k=2$, the same conclusion holds if $\operatorname{dim}_{\mathcal{H}}(E)>8 / 5$.

Now suppose that

$$
\operatorname{dim}_{\mathcal{H}}(E)>s_{k, d}:=\frac{d k}{k+1}
$$

Then $\mathcal{L}^{\left(\begin{array}{c}k+1 \\ 2\end{array}\right)-1}\left(S_{k}(E)\right)>0$.

Remark 1.6. In order to illustrate the extent to which the exponents in Theorem 1.5 improve on those in [8] and [7], consider the case $k=d$ where $s_{d, d}=$ $d-1+2 /(d+1)$. The exponent obtained in [8], [7] is $s_{d, d}^{\prime}=d-1 / 2+1 /(2 d)$. Note that $s_{d, d}<s_{d, d}^{\prime}$ for every $d \geq 2$, and, asymptotically as $d \rightarrow \infty$, the improvement is from $d-1 / 2$ to $d-1$. To put this in perspective, one has the following lower bound, which shows that for $k=d$ one cannot do better than $d-1$; for further discussion, see Section 4.

Remark 1.7. While the results of Theorem 1.5 significantly improve and extend the exponents in [4], [8], [7], the group-theoretic nature of our methods also casts new light upon the classical Mattila integral (see Section 5), potentially leading to further progress on related problems.

Theorem 1.8. Let $\alpha_{k, d}$ denote the optimal exponent for the congruent d-dimensional simplex problem, i.e., $\alpha_{k, d}$ is the infimum of those $\alpha$ for which $\mathcal{L}^{\left(\begin{array}{c}k+1 \\ 2\end{array}\right)}\left(T_{k}(E)\right)>0$ whenever $\operatorname{dim}_{\mathcal{H}}(E)>\alpha$. Then

$$
\alpha_{k, d} \geq \max \{k-1, d / 2\} .
$$

Moreover, $\alpha_{2,2} \geq 3 / 2$. 
We thank Ciprian Demeter for pointing out the relevance of [1] to the question of sharpness examples, and an anonymous referee for suggesting many improvements to the paper.

\section{Proofs of Theorem 1.3 and Theorem 1.4}

Motivated by the geometric viewpoint in [9], the essence of our approach is the following. Define a measure $d \nu$ on $\mathbb{R}^{\left(\begin{array}{c}k+1 \\ 2\end{array}\right)}=\mathbb{R}^{k(k+1) / 2}$, with support in $T_{k}(E)$, as in (1.2) above. We will show that to prove Theorem 1.3 it suffices to obtain an upper bound on the $L^{2}$ norm of the density, i.e., the Radon-Nikodym derivative of $d \nu$, which we denote by $\nu(\mathbf{t})$. We start by showing that

$$
\begin{aligned}
& \int \nu^{2}(\mathbf{t}) d \mathbf{t} \leq c_{k, d} \cdot \liminf _{\epsilon \rightarrow 0} \epsilon^{-k(k+1) / 2} \mu^{2(k+1)}\left\{\left(x^{1}, \ldots, x^{k+1}, y^{1}, \ldots, y^{k+1}\right)\right. \\
& \left.(2.1) \quad \in\left(\mathbb{R}^{d}\right)^{2(k+1)}:|| x^{i}-x^{j}|-| y^{i}-y^{j} \| \leq \epsilon, 1 \leq i<j \leq k+1\right\},
\end{aligned}
$$

where $\mu^{2(k+1)}$ denotes $\mu \times \cdots \times \mu, 2(k+1)$ times, with the proof showing that if the right-hand side of (2.1) is finite, then in fact $d \nu$ is absolutely continuous with respect to Lebesgue measure $d \mathbf{t}$, with density $\nu(\mathbf{t}) \in L^{2}$. Let $\phi \in$ $C_{0}^{\infty}\left(\mathbb{R}^{k(k+1) / 2}\right)$ such that $\phi \geq 0, \operatorname{supp}(\phi) \subset\{\mathbf{t} \mid \leq 1\}$, and $\int \phi d \mathbf{t}=1$, and let $\phi_{\epsilon}(\cdot)=\epsilon^{-k(k+1) / 2} \phi\left(\epsilon^{-1} \cdot\right)$, for $0<\epsilon<\infty$, be the resulting approximate identity. Setting $\nu_{\epsilon}=\phi_{\epsilon} * d \nu \in C_{0}^{\infty}$, one has $d \nu=w k^{*}-\lim _{\epsilon \rightarrow 0} \nu_{\epsilon}$, and (2.1) will follow if one shows that $\liminf _{\epsilon \rightarrow 0}\left\|\nu_{\epsilon}\right\|_{L^{2}}^{2}=C<\infty$.

Now,

$$
\nu_{\epsilon}(\mathbf{t})=\left\langle d \nu, \phi_{\epsilon}(\cdot-\mathbf{t})\right\rangle=\int \phi_{\epsilon}\left(\left(\left|x^{i}-x^{j}\right|-t_{i j}\right)_{1 \leq i<j \leq k+1}\right) d \mu\left(x^{1}\right) \cdots d \mu\left(x^{k+1}\right) .
$$

Due to the nonnegativity of $\phi_{\epsilon}$ and $d \mu$, this is dominated by

$$
\int \prod_{1 \leq i<j \leq k+1} \epsilon^{-1} \chi\left\{|| x^{i}-x^{j}\left|-t_{i j}\right|<\epsilon\right\} d \mu\left(x^{1}\right) \cdots d \mu\left(x^{k+1}\right),
$$

where $\chi_{A}(\cdot)$ denotes the characteristic function of a set $A$, and thus

$$
\begin{gathered}
\left\|\nu_{\epsilon}\right\|_{L^{2}}^{2} \lesssim \int_{1 \leq i<j \leq k+1} \prod_{1} \epsilon^{-1} \chi\left\{|| x^{i}-x^{j}\left|-t_{i j}\right|<\epsilon\right\} \prod_{1 \leq i<j \leq k+1} \epsilon^{-1} \chi\left\{|| y^{i}-y^{j}\left|-t_{i j}\right|<\epsilon\right\} \\
\cdot d \mu\left(x^{1}\right) \cdots d \mu\left(x^{k+1}\right) d \mu\left(y^{1}\right) \cdots d \mu\left(y^{k+1}\right) d \mathbf{t} .
\end{gathered}
$$

Now, by the triangle inequality, one has

$\chi\left\{|| x^{i}-x^{j}\left|-t_{i j}\right|<\epsilon\right\} \cdot \chi\left\{|| y^{i}-y^{j}\left|-t_{i j}\right|<\epsilon\right\} \leq \chi\left\{|| x^{i}-x^{j}|-| y^{i}-y^{j}||<2 \epsilon\right\}$, and thus, integrating out $d \mathbf{t}$, the right-hand side of (2.2) is

$$
\begin{aligned}
\lesssim \epsilon^{-k(k+1) / 2} \int \prod_{1 \leq i<j \leq k+1} \chi\left\{|| x^{i}-x^{j}|-| y^{i}-y^{j} \|<2 \epsilon\right\} \\
\cdot d \mu\left(x^{1}\right) \cdots d \mu\left(x^{k+1}\right) d \mu\left(y^{1}\right) \cdots d \mu\left(y^{k+1}\right) .
\end{aligned}
$$

Taking the liminf as $\epsilon \rightarrow 0$ yields the right-hand side of (2.1). 
To continue, we next introduce some notation. We write $\mathbf{x}:=\left(x^{1}, \ldots, x^{k+1}\right)$ for an ordered $(k+1)$-tuple of elements of $\mathbb{R}^{d}$. If the corresponding set $\left\{x^{1}, \ldots, x^{k+1}\right\}$ is nondegenerate (i.e., affinely independent), then

$$
\pi(\mathbf{x}):=\operatorname{span}\left\{x^{2}-x^{1}, \ldots, x^{k+1}-x^{1}\right\}
$$

is a $k$-dimensional linear subspace of $\mathbb{R}^{d}$. Let $\Delta(\mathbf{x})$ be the (unoriented) simplex generated by $\left\{x^{1}, \ldots, x^{k+1}\right\}$, i.e., the closed convex hull, which is contained in the affine plane $x^{1}+\pi(\mathbf{x})$. Both $\pi(\mathbf{x})$ and $\Delta(\mathbf{x})$ are independent of the order of the $x^{j}$. If $\left\{y^{1}, \ldots, y^{k+1}\right\}$ is congruent to $\left\{x^{1}, \ldots, x^{k+1}\right\}$, as defined in Definition 1.1 , then an elementary argument shows that, up to permutation of $y^{1}, \ldots, y^{k+1}$, there exists a $g \in \mathbb{O}(d)$ such that $x^{j}-x^{1}=g\left(y^{j}-y^{1}\right), 2 \leq j \leq k+1$, which is equivalent with $x^{j}-x^{i}=g\left(y^{j}-y^{i}\right), 1 \leq i<j \leq k+1$, and $\Delta(\mathbf{x})=\left(x^{1}-g y^{1}\right)+g \Delta(\mathbf{y})$. The group $\mathbb{O}(d)$ acts on the Grassmanians $G(k, d)$ and $G(d-k, d)$ of $k$ (respectively, $d-k$ ) dimensional linear subspaces of $\mathbb{R}^{d}$, and if $\mathbf{x}$ is congruent to $\mathbf{y}$, one has $\pi(\mathbf{x})=g \pi(\mathbf{y})$ and $\pi(\mathbf{x})^{\perp}=g\left(\pi(\mathbf{y})^{\perp}\right)$. The set of $g \in \mathbb{O}(d)$ fixing $\Delta(\mathbf{x})$ is a conjugate of $\mathbb{O}(d-k) \subset \mathbb{O}(d)$, and we refer to this as the stabilizer of $\Delta(\mathbf{x})$, denoted $\operatorname{Stab}(\mathbf{x})$.

For $\mathbf{x}, \mathbf{y}$ congruent as above, let $\tilde{g} \in \mathbb{O}(d)$ be such that $\pi(\mathbf{x})=\tilde{g} \pi(\mathbf{y})$. Then, $x^{i}-x^{j}=\tilde{g} h\left(y^{i}-y^{j}\right)$ for all $h \in \operatorname{Stab}(\mathbf{y})$. For each $\mathbf{y}$, take a cover of $\mathbb{O}(d) / \operatorname{Stab}(\mathbf{y})$ by balls of radius $\epsilon$ (with respect to some Riemannian metric) with finite overlap. Since the dimension of $\mathbb{O}(d) / \operatorname{Stab}(\mathbf{y})$ is that of $\mathbb{O}(d) / \mathbb{O}(d-k)$, namely

$$
\frac{d(d-1)}{2}-\frac{(d-k)(d-k-1)}{2}=k d-\frac{k(k+1)}{2},
$$

we need $N(\epsilon) \sim C \epsilon^{-(k d-k(k+1) / 2)}$ balls to cover it. Choose sample points, $\tilde{g}_{m}(\mathbf{y})$, $1 \leq m \leq N(\epsilon)$, one in each of the balls.

From basic geometry one sees that the set

$$
\left\{(\mathbf{x}, \mathbf{y}):|| x^{i}-x^{j}|-| y^{i}-y^{j}|| \leq \epsilon, 1 \leq i<j \leq k+1\right\}
$$

is contained in

$\bigcup_{m=1}^{N(\epsilon)}\left\{(\mathbf{x}, \mathbf{y}):\left|\left(x^{i}-x^{j}\right)-\tilde{g}_{m}(\mathbf{y}) h\left(y^{i}-y^{j}\right)\right| \leq C \epsilon, \forall 1 \leq i<j \leq k+1, h \in \operatorname{Stab}(\mathbf{y})\right\}$,

where $C=2 \max \{\operatorname{diam}(E), 1\}$. Thus, the expression within the liminf on the right-hand side of (2.1) is bounded above by

$$
\begin{aligned}
& \epsilon^{-k(k+1) / 2} \sum_{m=1}^{N(\epsilon)} \mu^{2(k+1)}\left\{(\mathbf{x}, \mathbf{y}):\left|\left(x^{i}-x^{j}\right)-\tilde{g}_{m}(\mathbf{y}) h\left(y^{i}-y^{j}\right)\right| \leq C \epsilon,\right. \\
&\forall 1 \leq i<j \leq k+1, h \in \operatorname{Stab}(\mathbf{y})\},
\end{aligned}
$$

which can also be written as

$$
\begin{array}{r}
\epsilon^{-k d} \sum_{m=1}^{N(\epsilon)} \epsilon^{k d-k(k+1) / 2} \mu^{2(k+1)}\left\{(\mathbf{x}, \mathbf{y}):\left|\left(x^{i}-\tilde{g}_{m}(\mathbf{y}) h y^{i}\right)-\left(x^{j}-\tilde{g}_{m}(\mathbf{y}) h y^{j}\right)\right| \leq C \epsilon,\right. \\
(2.3) \quad \forall 1 \leq i<j \leq k+1, h \in \operatorname{Stab}(\mathbf{y})\} .
\end{array}
$$


Since this holds for any choice of sample points $\tilde{g}_{m}(\mathbf{y})$, we can pick these points such that they minimize (up to a factor of $1 / 2$, say) the quantity

$$
\begin{aligned}
\mu^{2(k+1)}\left\{(\mathbf{x}, \mathbf{y}):\left|\left(x^{i}-\tilde{g}_{m}(\mathbf{y}) h y^{i}\right)-\left(x^{j}-\tilde{g}_{m}(\mathbf{y}) h y^{j}\right)\right| \leq \epsilon,\right. \\
\forall 1 \leq i<j \leq k+1, h \in \operatorname{Stab}(\mathbf{y})\} .
\end{aligned}
$$

Now consider the $N(\epsilon)$ preimages, under the natural projection from $\mathbb{O}(d)$, of the balls used to cover $\mathbb{O}(d) / \operatorname{Stab}(\mathbf{y})$; we can label these $\epsilon$-tubular neighborhoods of the preimages of the sample points $\tilde{g}_{m}(\mathbf{y})$ as $T_{1}^{\epsilon}, \ldots, T_{N(\epsilon)}^{\epsilon}$. Since $\operatorname{dim}(\mathbb{O}(d) / \operatorname{Stab}(\mathbf{y}))=k d-k(k+1) / 2$, each $T_{m}^{\epsilon}$ has volume $\sim \epsilon^{k d-k(k+1) / 2}$. The infimum over a set is less than or equal to the average over the set, so we obtain

$$
\begin{array}{r}
\mu^{2(k+1)}\left\{(\mathbf{x}, \mathbf{y}):\left|\left(x^{i}-\tilde{g}_{m}(\mathbf{y}) h y^{i}\right)-\left(x^{j}-\tilde{g}_{m}(\mathbf{y}) h y^{j}\right)\right| \leq \epsilon,\right. \\
\forall 1 \leq i<j \leq k+1, h \in \operatorname{Stab}(\mathbf{y})\} \\
\lesssim \frac{1}{\epsilon^{k d-k(k+1) / 2}} \int_{T_{m}^{\epsilon}} \mu^{2(k+1)}\left\{(\mathbf{x}, \mathbf{y}):\left|\left(x^{i}-g y^{i}\right)-\left(x^{j}-g y^{j}\right)\right| \leq \epsilon,\right. \\
1 \leq i<j \leq k+1\} d g .
\end{array}
$$

We can thus bound (2.3) above by

$$
\epsilon^{-k d} \sum_{m=1}^{N(\epsilon)} \int_{T_{m}^{\epsilon}} \mu^{2(k+1)}\left\{(\mathbf{x}, \mathbf{y}):\left|\left(x^{i}-g y^{i}\right)-\left(x^{j}-g y^{j}\right)\right| \leq \epsilon, 1 \leq i<j \leq k+1\right\} d g .
$$

Since the cover has finite overlap, this in turn can be bounded above, up to a constant $c_{k, d}$, by

$$
\epsilon^{-k d} \int \mu^{2(k+1)}\left\{(\mathbf{x}, \mathbf{y}):\left|\left(x^{i}-g y^{i}\right)-\left(x^{j}-g y^{j}\right)\right| \leq \epsilon, 1 \leq i<j \leq k+1\right\} d g,
$$

and taking the liming, we obtain a constant multiple of the expression (1.3). This completes the proof of Theorem 1.3.

If one studies similar simplices instead of congruent ones, letting $S_{k}(E)$ as in Definition 1.2, then the preceding analysis goes through essentially unchanged, except that in place of (1.3) we have (1.5). This establishes Theorem 1.4.

\section{Proof of Theorem 1.5}

The matters have been reduced in the introduction to the estimation of (1.3). We shall need the following result.

Theorem 3.1. Let $\mu$ be a compactly supported Borel measure. Then, for $s \geq d / 2$, and $\epsilon>0$,

$$
\int_{S^{d-1}}|\widehat{\mu}(t \omega)|^{2} d \omega \leq C_{\epsilon} I_{s}(\mu) t^{\epsilon-\gamma_{s}}
$$

with $\gamma_{s}=(d+2 s-2) / 4$ if $d / 2 \leq s \leq(d+2) / 2$, and $\gamma_{s}=s-1$ for $s \geq(d+2) / 2$.

For $s \leq(d+2) / 2$, this is due to Wolff [15] $(d=2)$ and Erdog̃an [3] $(d \geq 3)$; the easier case of $s \geq(d+2) / 2$ is due to Sjölin [14]. 
As we note above, the proof of Theorem 1.5 is reduced to the verification of (1.3). Let $\psi$ be a smooth cutoff function supported in $\left\{\xi \in \mathbb{R}^{d}: 1 / 2 \leq|\xi| \leq 4\right\}$ and identically equal to 1 in $\left\{\xi \in \mathbb{R}^{d}: 1 \leq|\xi| \leq 2\right\}$. Let $\nu_{g, j}$ denote the $j$ th Littlewood-Paley piece of $\nu_{g}$, defined by the relation $\widehat{\nu}_{g, j}(\xi)=\widehat{\nu}_{g}(\xi) \psi\left(2^{-j} \xi\right)$. Since $\nu_{g}$ is compactly supported, we may assume that $j \geq 0$. Using the LittlewoodPaley decomposition of $\nu_{g}$, the integral in (1.3) equals

$$
\int \sum_{j_{1}, \ldots, j_{k+1}} \nu_{g, j_{1}}(x) \nu_{g, j_{2}}(x) \cdots \nu_{g, j_{k+1}}(x) d x .
$$

We can split this sum up into $k(k+1)$ sums of the type where we sum up over indices where $j_{1} \geq j_{2} \geq j_{3}, \ldots, j_{k+1}$ and permutations thereof. It suffices to show bounds for one of those sums so without loss of generality we may assume we are in the case $j_{1} \geq j_{2} \geq j_{3}, \ldots, j_{k+1}$. Passing to the Fourier side we can write

$$
\begin{aligned}
\int \sum_{j_{1} \geq j_{2} \geq j_{3} \ldots, j_{k+1}} \nu_{g, j_{1}}(x) \nu_{g, j_{2}}(x) \cdots \nu_{g, j_{k+1}}(x) d x \\
=\sum_{j_{1} \geq j_{2} \geq j_{3} \ldots, j_{k+1}} \int \widehat{\nu}_{g, j_{1}} * \widehat{\nu}_{g, j_{3}} * \cdots * \widehat{\nu}_{g, j_{k+1}}(\xi) \cdot \widehat{\nu}_{g, j_{2}}(\xi) d \xi .
\end{aligned}
$$

Now $\widehat{\nu}_{g, j_{1}} * \widehat{\nu}_{g, j_{3}} * \cdots * \widehat{\nu}_{g, j_{k+1}}$ is supported on scale $2^{j_{1}}+2^{j_{3}}+\cdots+2^{j_{k+1}} \sim 2^{j_{1}}$, while $\widehat{\nu}_{g, j_{2}}$ is supported on scale $2^{j_{2}}$, so by Plancherel it is clear that the sum vanishes if $j_{1}-j_{2}>2$. Thus it suffices to consider the case $j_{1}=j_{2}$ and to study

$$
\sum_{j_{1}=j_{2} \geq j_{3} \ldots, j_{k+1}} \int \nu_{g, j_{1}}(x) \nu_{g, j_{2}}(x) \cdots \nu_{g, j_{k+1}}(x) d x .
$$

As above, $\nu_{g, j}(x)=\mu_{j} * \mu_{j}(g \cdot)$, so

$$
\left\|\nu_{g, j}\right\|_{\infty} \leq\left\|\mu_{j}\right\|_{1} \cdot\left\|\mu_{j}\right\|_{\infty} \leq C 2^{j(d-s)}
$$

for any $s<\operatorname{dim}_{\mathcal{H}}(E)$, since $\mu$ is a Frostman measure supported on $E$ (see, e.g., Chapter 8 in [12]). To see this, observe that $\left\|\mu_{j}\right\|_{1} \leq 1$ trivially since $\mu$ is a probability measure and

$$
\left|\mu_{j}(x)\right|=2^{d j}\left|\mu * \widehat{\psi}\left(2^{j} \cdot\right)(x)\right| \leq C_{N} 2^{d j} \int\left(1+2^{j}|x-y|\right)^{-N} d \mu(y) \leq C_{N}^{\prime} 2^{j(d-s)}
$$

since $\mu$ is a Frostman measure on $E$. Using this estimate on the terms corresponding to the indices $j_{3}, \ldots, j_{k+1}$ we can bound (3.1) above, up to a fixed constant, by

$$
\sum_{j}\left(\sum_{j_{3}, \ldots, j_{k+1} \leq j} 2^{\left(j_{3}+\cdots+j_{k+1}\right)(d-s)}\right) \nu_{g, j}^{2}(x) \lesssim \sum_{j} 2^{j(k-1)(d-s)} \nu_{g, j}^{2}(x) .
$$

It follows that we can bound (1.3) by a finite sum of terms of the type

$$
\sum_{j} 2^{j(k-1)(d-s)} \cdot \iint \nu_{g, j}^{2}(x) d x d g .
$$


By Plancherel (see the discussion in Section 5 below),

$$
\iint \nu_{g, j}^{2}(x) d x d g \approx \int_{2^{j}}^{2^{j+1}}\left(\int_{S^{d-1}}|\widehat{\mu}(t \omega)|^{2} d \omega\right)^{2} t^{d-1} d t \leq C^{\prime \prime} 2^{j(d-s)} 2^{-j \gamma(s, d)},
$$

with the inequality following from Theorem 3.1.

Let us first handle the case $d \geq 3$. Inserting the last inequality back into (3.2), we see that geometric series converges if $(d-s) k-(s-1)<0$, which yields the condition $s>(d k+1) /(k+1)$, as claimed. If $d=k=2, \gamma(s, 2)=s / 2$ and the geometric series converges if $s>8 / 5$. This completes the proof of the first part of Theorem 1.5.

To prove the second part, as explained in Section 2, it suffices to estimate (1.5). Following the proof of the first part of Theorem 1.5 above, the second part would follow from the estimate

$$
\left.\left.\left|\int_{1}^{2} \int\right| \widehat{\mu}(a g \xi)\right|^{2} d g \frac{d a}{a}|\leq C| \xi\right|^{-s},
$$

where the reduction to $a \in[1,2]$ is accomplished by simple pigeon-holing and scaling. Indeed, recall that $\mu$ is a Frostman measure supported on $E$ (see, e.g., Chapter 8 in [16], ) means that for any $\epsilon>0$ there exists $C_{\epsilon}>0$ such that if $B_{\delta}$ is a ball of radius $\delta$ centered at the origin, then

$$
\mu\left(B_{\delta}\right) \leq C_{\epsilon} \delta^{s-\epsilon},
$$

where $s$ is the Hausdorff dimension of $E$ and $\epsilon>0$ is arbitrarily small. If $\delta$ is sufficiently small, this quantity is $<1 / 2$, so the intersection $E$ and the complement of $B_{\delta}$ has $\mu$-measure $>1 / 2$. Renaming this intersection as $E$ and rescaling, the procedure that does not affect whether the Lebesgue measure of $T_{k}(E)$ (or $\left.S_{k}(E)\right)$ is positive, we achieve the desired setup.

By the action of the orthogonal group on the sphere, (3.3) would follow from

$$
\left.\left.\left|\int_{1}^{2} \int_{S^{d-1}}\right| \widehat{\mu}(a|\xi| \omega)\right|^{2} d \omega \frac{d a}{a}|\leq C| \xi\right|^{-s}
$$

for any $s<\operatorname{dim}_{\mathcal{H}}(E)$. This in turn is proven by observing that

$$
\begin{aligned}
& \int_{1}^{2} \int_{S^{d-1}}|\widehat{\mu}(a|\xi| \omega)|^{2} d \omega \frac{d a}{a}=\int_{|\xi|}^{2|\xi|} \int_{S^{d-1}}|\widehat{\mu}(a \omega)|^{2} d \omega \frac{d a}{a} \\
& \leq|\xi|^{-d} \int_{|\xi|}^{2|\xi|} \int_{S^{d-1}}|\widehat{\mu}(a \omega)|^{2} d \omega a^{d-1} d a=|\xi|^{-d} \int_{|\xi| \leq|x| \leq 2|\xi|}|\widehat{\mu}(x)|^{2} d x \leq C|\xi|^{-s} .
\end{aligned}
$$

\section{Sharpness of lower bounds}

We now turn to the proof of Theorem 1.8. If $E$ is contained in a (k-1)-dimensional plane, every simplex with $k+1$ points in $E$ is degenerate, so the restriction $\alpha_{k, d}>k-1$ is clear. The lower bound $\alpha_{k, d}>d / 2$ is also necessary, since this threshold is needed to ensure that $\mathcal{L}^{1}(\Delta(E))>0$ for general $E$, as was noted in the introduction. 
The restriction $\alpha_{2,2}>3 / 2$ follows from a lattice construction and simple number-theoretic analysis, given below but previously obtained by Burak Erdog̃an and the second listed author [5]. Start by considering the following construction in $\mathbb{R}^{d}$ for general $d \geq 1$. Let $q_{1}=2$ and recursively choose $q_{i} \in \mathbb{N}$ with $q_{i+1}>q_{i}^{i}, \forall i \geq 1$. Fix $s<d$ and let $E_{i}$ denote the $q_{i}^{-d / s}$-neighborhood of $\mathbb{Z}^{d} \cap\left[0, q_{i}\right]^{d}$, scaled by $q_{i}^{-1}$ so as to be a subset of $[0,1]^{d}$. Define $E=\cap_{i} E_{i}$. The proof that $\operatorname{dim}_{\mathcal{H}}(E)=s$ can be found in Chapter 8 of [6] in the case of $d=1$. The higher dimensional argument follows from the same argument.

Now let $d=2$. To show that $\alpha_{2,2} \geq 3 / 2$, let $q=q_{i}$, for $i$ very large. We claim that

$$
\mathcal{L}^{3}\left(T_{2}\left(E_{q}\right)\right) \lesssim q^{-6 / s} \cdot \# T_{2}\left(\left\{\mathbb{Z}^{2} \cap[0, q]^{2}\right\}\right) \leq C_{\epsilon} q^{-6 / s} \cdot q^{4+\epsilon}
$$

In fact, note that, by translation invariance, in order to count congruence classes determined by the unrescaled $\mathbb{Z}^{2} \cap[0, q]^{2}$ it is enough to place one vertex at the origin. Call the remaining vertices $v$ and $w$ and let $|v|=a,|w|=b$. For our purposes it is sufficient to know that the number of choices is $\leq C q^{2}$. To see this simply observe that squares of the distances from the origin are integers in $\left[0,2 q^{2}\right]$, so there cannot possibly be more than $2 q^{2}$ of them. Once $|v|$ and $|w|$ are fixed, it remains to compute how many possibilities there are for $|v-w|$. This number cannot exceed the product of the number of integer points on $\{x:|x|=a\}$ and the number of integer points on $\{x:|x|=b\}$. It is well known that for any $\epsilon>0$, the number of lattice points on the circle of radius $r$ in the plane does not exceed $C_{\epsilon} r^{\epsilon}$; see, e.g., [10]. The estimate (4.1) thus follows and we conclude that $\mathcal{L}^{3}\left(T_{2}(E)\right)$ is not in general positive if the Hausdorff dimension of $E$ is smaller than $3 / 2$.

Note that this argument does not show what happens at $s=3 / 2$.

Examination of $\alpha_{3,3}$ leads to an interesting lattice point problem. Take $d=3$ in the construction above. Once again, we place one vertex at the origin and call the remaining vertices $v^{1}, v^{2}, v^{3}$. There are $\approx q^{2}$ choices for $\left|v^{i}\right|$. It remains to count the number of non-congruent configurations that $v^{i} \mathrm{~s}$ can form. Each $v^{i}$ lies on a sphere of radius at most $q$ and it is well-known that the number of lattice points a sphere of radius $r$ in $\mathbb{R}^{3}$ is $\lesssim q$. It follows by trivial counting that the number of non-congruent configurations of $v^{j}$ is $\lesssim q^{3}$. We deduce that

$$
\mathcal{L}^{6}\left(T_{4}\left(E_{q}\right)\right) \lesssim q^{-18 / s} \cdot q^{6} \cdot q^{3}
$$

which results in the trivial restriction $s>2$. So the question of whether we can obtain a tighter restriction on $\operatorname{dim}_{\mathcal{H}}(E)$, needed to ensure that $\mathcal{L}^{6}\left(T_{4}(E)\right)>0$, comes down to estimating the size of the discrete set

$$
T_{3}\left(S_{1} \cap \mathbb{Z}^{3}, S_{2} \cap \mathbb{Z}^{3}, S_{3} \cap \mathbb{Z}^{3}\right),
$$

the number of non-congruent triangles with vertices at lattice points on spheres $S_{1}, S_{2}, S_{3}$ of radii $\approx q$. Any estimate of the form

$$
\# T_{3}\left(S_{1} \cap \mathbb{Z}^{3}, S_{2} \cap \mathbb{Z}^{3}, S_{3} \cap \mathbb{Z}^{3}\right) \leq C q^{3-\delta}
$$


for some $\delta>0$ would immediately allow one to conclude that

$$
\alpha_{3,3} \geq 2+\delta^{\prime}
$$

for some $\delta^{\prime}>0$. We do not know whether (4.2) holds, and pose this question as an open problem that is interesting in its own right. ${ }^{1}$

\section{A stationary phase-free proof of the $(d+1) / 2$ exponent in the Falconer problem}

The purpose of this section is to make a couple of simple observations regarding the Falconer distance conjecture and the methods of proof that have been employed to attack it. First, we apply the results of Section 3 to the case $k=1$, corresponding to the Falconer distance problem. Applying (1.1) with $f(z)=e^{-2 \pi i z \cdot \xi}$, we obtain

$$
\widehat{\nu}_{g}(\xi)=\widehat{\mu}(\xi) \widehat{\mu}(g \xi),
$$

which means that, via Plancherel, the expression in (1.3), with $k=1$ is equal to

$$
\int_{\mathbb{R}^{d}}|\widehat{\mu}(\xi)|^{2}\left\{\int_{\mathbb{O}(d)}|\widehat{\mu}(g \xi)|^{2} d g\right\} d \xi .
$$

A moment's reflection shows that this quantity equals a constant multiple of

$$
\int\left(\int_{S^{d-1}}|\widehat{\mu}(t \omega)|^{2} d \omega\right)^{2} t^{d-1} d t,
$$

the classical Mattila integral derived in [11], which has so far been the main tool in the study of the Falconer distance problem. The fact that the boundedness of this integral implies a lower bound on the Lebesgue measure of the distance set is typically derived using the method of stationary phase (see also Chapter 9 of [16]), but the argument above shows that a group-theoretic argument can be used instead.

We now establish the fact that the threshold $(d+1) / 2$ for the Falconer distance conjecture can be established using our geometric methods without the use of the method of stationary phase. See also Mitsis [13] for another geometric argument in the context of the Falconer distance problem.

The argument culminating in (5.1) above, reproves the classical result due to Mattila, namely that if $E$ is a compact subset of $\mathbb{R}^{d}$ of Hausdorff dimension $s>d / 2$, and the Mattila integral given by (5.1) is bounded for some Borel measure $\mu$ supported on $E$, then the Lebesgue measure of $\Delta(E)$ is positive. The fact that the expression in (5.1) is bounded if the Hausdorff dimension of $E$ is greater than $(d+1) / 2$ for any Frostman measure $\mu$ supported on $E$ (see [12], p. 112, for background on Frostman's lemma) follows immediately from the following simple observation. Recall the $s$-energy integral of $\mu$,

$$
I_{s}(\mu)=\iint|x-y|^{-s} d \mu(x) d \mu(y) .
$$

\footnotetext{
${ }^{1}$ After submission of this paper, this question was answered in the negative by Demeter [2].
} 
Lemma 5.1 ([16], p.61). Let $\mu$ be a compactly supported Borel measure on $\mathbb{R}^{d}$, $d \geq 2$. Then, for any $s \geq d / 2$,

$$
\int_{S^{d-1}}|\widehat{\mu}(t \omega)|^{2} d \omega \leq C I_{s}(\mu) t^{-(s-1)}, \quad 0<t<\infty .
$$

We give the proof of Lemma 5.1 for the sake of completeness. Let $\phi$ be a radial smooth function with compact support whose Fourier transform is $\geq 1$ on the support of $\mu$. Then it suffices to estimate

$$
\begin{aligned}
\int|\phi * \hat{\mu}(t \omega)|^{2} d \omega & \leq\left(\int\left(\int|\phi(x-t \omega)|^{2}|\widehat{\mu}(x)|^{2} d \omega\right)^{1 / 2} d x\right)^{2} \\
& \leq C^{\prime} t^{-(d-1)} \int_{|| x|-t| \leq C^{\prime \prime}}|\widehat{\mu}(x)|^{2} d x \leq C^{\prime \prime \prime} I_{s}(\mu) t^{-s+1}
\end{aligned}
$$

finishing the proof.

To see how Lemma 5.1 implies the $(d+1) / 2$ exponent for the Falconer problem, it is enough to prove that the Mattila integral (5.1) is bounded if $\mu$ is a Borel measure supported on a set of Hausdorff dimension greater than $(d+1) / 2$. Using Lemma 5.1 we see that (with $I_{s}(\mu)$ as in $(5.2)$ above)

$$
\begin{aligned}
\int\left(\int_{S^{d-1}}|\widehat{\mu}(t \omega)|^{2} d \omega\right)^{2} t^{d-1} d t & \leq C \iint t^{d-1} t^{-s+1}|\widehat{\mu}(t \omega)|^{2} d \omega d t \\
& =C \int|\widehat{\mu}(\xi)|^{2}|\xi|^{-s+1} d \xi \\
& =C \int|\widehat{\mu}(\xi)|^{2}|\xi|^{-d+(d-s+1)} d \xi \leq C^{\prime \prime} I_{s}(\mu)
\end{aligned}
$$

if $s>(d+1) / 2$, as desired.

\section{References}

[1] Bourgain, J. And Demeter, C.: New bounds for the discrete Fourier restriction to the sphere in 4D and 5D. Int. Math. Res. Not. 2015 (2015), no. 11, 3150-3184.

[2] Demeter, C.: On the number of non-congruent lattice tetrahedra. Preprint available at http://arxiv.org/abs/1312.1714, 2013.

[3] ERdoĞAN, B.: A bilinear Fourier extension theorem and applications to the distance set problem. Int. Math. Res. Not. 2005, no. 23, 1411-1425.

[4] ErdoĞan, B., Hart, D. And Iosevich, A.: Multiparameter projection theorems with applications to sums-products and finite point configurations in the Euclidean setting. In Recent advances in harmonic analysis and applications, 93-103. Springer Proc. Math. Stat. 25, Springer, New York, 2013.

[5] ErdoĞan, B. And Iosevich, A.: Conversation in a coffee shop in Vancouver, 2011.

[6] Falconer, K. J.: On the Hausdorff dimensions of distance sets. Mathematika 32 (1985), no. 2, 206-212 (1986). 
[7] Grafakos, L., Greenleaf, A., Iosevich, A. and Palsson, E.: Multilinear generalized Radon transforms and point configurations. Forum Math. 27 (2015), no. 4, 2323-2360.

[8] Greenleaf, A. And Iosevich, A.: On triangles determined by subsets of the Euclidean plane, the associated bilinear operators and applications to discrete geometry. Anal. PDE 5 (2012), no. 2, 397-409.

[9] Iosevich, A. And Liu, B.: The structure of $S L_{d}(\mathbb{R})$ and the dot product variant of the Falconer distance conjecture. In preparation, 2013.

[10] Landau, E.: Vorlesungen über Zahlentheorie. Vol. I, Part 2; Vol. II; Vol. III (German). Chelsea Publ. Co., New York, 1969.

[11] Mattila, P.: Spherical averages of Fourier transforms of measures with finite energy; dimension of intersections and distance sets. Mathematika 34 (1987), 207-228.

[12] Mattila, P.: Geometry of sets and measures in Euclidean spaces. Cambridge Studies in Advanced Mathematics 44, Cambridge University Press, Cambridge, 1995.

[13] Mitsis, T.: A note on the distance set problem in the plane. Proc. Amer. Math. Soc. 130 (2002), no. 6, 1669-1672.

[14] SJöLIN, P.: Estimates of spherical averages of Fourier transforms and dimensions of sets. Mathematika 40 (1993), 322-330.

[15] WolfF, T.: Decay of circular means of Fourier transforms of measures. Int. Math. Res. Not. 1999, no. 10, 547-567.

[16] Wolff, T.: Lectures on harmonic analysis. University Lecture Series 29, American Mathematical Society, Providence, RI, 2003.

Received June 23, 2013; revised March 21, 2014.

Allan Greenleaf: Department of Mathematics, University of Rochester, Rochester, NY 14627, USA.

E-mail: allan@math.rochester.edu

Alex Iosevich: Department of Mathematics, University of Rochester, Rochester, NY 14627, USA.

E-mail: iosevich@math.rochester.edu

Bochen Liu: Department of Mathematics, University of Rochester, Rochester, NY 14627, USA.

E-mail: bliu19@z.rochester.edu

Eyvindur Palsson: Department of Mathematics and Statistics, Williams College, Williamstown, MA 01267, USA.

E-mail: eap2@williams.edu

The first two authors were partially supported by NSF Grants DMS-0853892 and DMS1045404, respectively. 